\title{
The association between age at menarche and later risk of gestational diabetes is mediated by insulin resistance
}

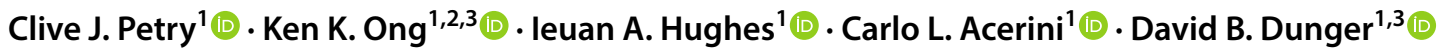

Received: 8 March 2018 / Accepted: 15 May 2018 / Published online: 22 May 2018

(c) The Author(s) 2018

\begin{abstract}
Aims Associations have been reported between age at menarche and the later risk of gestational diabetes. However, it is not known whether these associations reflect differences in insulin sensitivity and/or pancreatic $\beta$-cell function in pregnancy.

Methods We examined this question in women enrolled in the prospective Cambridge Baby Growth Study who recalled their age at menarche in questionnaires during pregnancy. Polynomial logistic and linear regression models were used to relate menarche timing to the risk of gestational diabetes, both unadjusted and adjusted for the Homeostasis Model Assessments of insulin resistance (HOMA IR) and pancreatic $\beta$-cell function (HOMA B) at week 28 of pregnancy.

Results Age at menarche showed a U-shaped association with gestational diabetes risk (linear term: $p=9.5 \times 10^{-4}$; quadratic term: $p=1.0 \times 10^{-3} ; n=889$; overall model $\left.p=8.1 \times 10^{-3}\right)$. Age at menarche showed a negative linear association with insulin resistance (HOMA IR: $\beta=-0.13, p=5.2 \times 10^{-4}, n=771$ ), which explained the relationship between age at menarche and gestational diabetes risk (adjusted linear term going from $p=0.03-0.08$; adjusted quadratic term going from $p=0.04-0.08$; $n=771$ ). Age at menarche also showed a negative linear association with $\beta$-cell function (HOMA B: $\beta=-0.11, p=2.8 \times 10^{-3}$, $n=771$ ) but this did not attenuate the relationship between age at menarche and gestational diabetes (adjusted linear term $p=0.02$; adjusted quadratic term $p=0.03, n=771$ ).

Conclusions These results suggest that the associations between age at menarche and risk of gestational diabetes and raised pregnancy glucose concentrations may be mediated by insulin resistance.
\end{abstract}

Keywords Puberty $\cdot$ Pregnancy $\cdot$ Insulin sensitivity $\cdot$ Adiposity $\cdot$ PAPP-A

Managed by Massimo Porta.

Electronic supplementary material The online version of this article (https://doi.org/10.1007/s00592-018-1162-7) contains supplementary material, which is available to authorized users.

Clive J. Petry

cjp1002@cam.ac.uk

1 Department of Paediatrics, University of Cambridge, Cambridge Biomedical Campus, Hills Road, Box 116, Cambridge CB2 0QQ, UK

2 Medical Research Council Epidemiology Unit, University of Cambridge, Cambridge CB2 0QQ, UK

3 The Institute of Metabolic Science, University of Cambridge, Cambridge CB2 0QQ, UK

\section{Introduction}

Many settings have observed secular trends towards a lowering of average age at menarche (AAM) in the last 50 years, coincident with higher levels of nutrition [1]. The AAM appears to be dependent on both genetic and environmental factors [2]. The trend towards its lowering is thought to be related to the improved socioeconomic conditions and the higher prevalence of childhood obesity [3]. Possibly through reflecting differences in the reproductive hormonal milieu, changes in AAM may be important since it appears to be related to health in adult life through associations with risk of adult obesity [4], type 2 diabetes [5], asthma [6], endometriosis [7], breast cancer [8] and death (by all causes [9]).

Not surprisingly given the strong links between type 2 diabetes and gestational diabetes (GDM) and the association between AAM and type 2 diabetes, recently AAM has also been found to be associated with the future risk of GDM in pregnancy [10-13]. As long ago as 1975 it 
was suggested that early menarche may be linked to GDM [14]. In each of the studies the highest risk of GDM was in women with the earliest AAM. However, this association has not been consistently found across all populations [15]. In the studies where an association between AAM and risk of future GDM was observed, no attempt was made to relate AAM to the principal mechanisms of glucose regulation: insulin sensitivity and secretion in pregnancy. In the present analysis we therefore tested whether AAM was associated with future GDM risk and raised glucose concentrations in pregnancy, and how such associations relate to changes in indices of insulin sensitivity and pancreatic $\beta$-cell function/insulin secretion in the Cambridge Baby Growth Study. Early pregnancy-associated plasma protein A (PAPP-A) concentrations were also assessed in relation to AAM since these are potential biomarkers of GDM risk [16] that appear to relate to changes in insulin sensitivity in pregnancy [17].

\section{Materials and methods}

\section{Cohort}

The prospective Cambridge Baby Growth Study recruited 2,229 women (and consequently their partners and babies) attending ultrasound clinics during early pregnancy at the Rosie Maternity Hospital, Cambridge, United Kingdom, between 2001 and 2009 [17, 18]. All study participants were over 16 years of age. At around 15 weeks of gestation (range 12-18 weeks) the mothers had a blood sample collected for the measurement of serum PAPP-A [17]. At around 28 weeks of gestation the mothers without known diabetes underwent a $75 \mathrm{~g}$ oral glucose tolerance test (OGTT) after fasting overnight. Venous blood was collected just prior to and $60 \mathrm{~min}$. (and $120 \mathrm{~min}$. after 2007) after the consumption of the glucose load for the measurement of plasma glucose, insulin and C-peptide concentrations. Capillary blood glucose measurements were made at $0,30,60,90$, and $120 \mathrm{~min}$. using an Abbott Freestyle Mini (Abbott Diagnostics, Maidenhead, UK). GDM, with a prevalence of $10.2 \%$ in the whole cohort, was defined here using the International Association of Diabetes in Pregnancy Study Groups thresholds [19] using the 0 and $60 \mathrm{~min}$. venous plasma glucose concentrations as described [20].

Each study participant was given a printed questionnaire at recruitment to fill in and return once the pregnancy was completed. One of the questions asked "What age were you when you had your first period?" which the study participants tended to answer in terms of whole years. A total of 1273 women $(57.1 \%)$ filled in and returned their questionnaires.

\section{Ethics}

The Cambridge Baby Growth Study was approved by the local ethics committee, Addenbrooke's Hospital, Cambridge, United Kingdom. All procedures followed were in accordance with the institutional guidelines and therefore the ethical standards as laid down in the 1964 Declaration of Helsinki and its later amendments. Written informed consent was obtained from all the study participants prior to their inclusion in the study.

\section{Laboratory measurements}

Serum PAPP-A was measured using a time-resolved fluorescence immunoassay as described previously [17]. Venous plasma glucose concentrations were measured using a routine glucose oxidase-based method. Plasma C-peptide and insulin concentrations were measured using Diagnostic System Laboratories (London, UK) ELISAs run according to the manufacturer's instructions. The sensitivity of the C-peptide assay was $0.004 \mathrm{nmol} / \mathrm{L}$. Its intra-assay $\mathrm{CV}$ was $2.4 \%$ at $3.1 \mathrm{nmol} / \mathrm{L}$ and its equivalent inter-assay $\mathrm{CV}$ was $2.7 \%$. The sensitivity of the insulin assay was $2 \mathrm{pmol} / \mathrm{L}$. Its intra-assay $\mathrm{CV}$ was $4.4 \%$ at $72 \mathrm{pmol} / \mathrm{L}$ and its equivalent inter-assay CV was $8.7 \%$.

\section{Calculations}

The body mass index (BMI) was calculated as the selfreported pre-pregnancy body weight divided by the height squared. Insulin sensitivity and pancreatic $\beta$-cell function (insulin secretion) were estimated using the Homeostasis Model Assessment (HOMA), calculated using the week 28 circulating glucose and C-peptide concentrations using the online HOMA calculator [21]. Insulin secretion was quantified as the C-peptidogenic index, calculated as (C-peptide $60 \mathrm{~min}-\mathrm{C}$-peptide $0 \mathrm{~min}) /($ glucose $60 \mathrm{~min}$-glucose $0 \mathrm{~min})$. The insulin secretion for the given insulin sensitivity was assessed in terms of the C-peptide disposition index, calculated as the $\mathrm{C}$-peptidogenic index divided by the fasting $\mathrm{C}$-peptide concentration. Equivalent indices were calculated using insulin instead of C-peptide concentrations but C-peptide-derived variables were used in the statistical models so that they were not affected by hepatic insulin extraction. The areas under the OGTT capillary whole blood glucose curves (AUC) were calculated using the trapezoid rule.

\section{Statistical analyses}

The association between AAM and GDM was analysed using logistic regression, both unadjusted and adjusted for 
covariates. Polynomial logistic regression was also used to test for a non-linear association as reported by Li et al. [12] and also apparent in other studies $[11,13,15]$. In our analysis the GDM risk was fitted using Stata's qfit function, having already tested whether or not there was a linear relationship with AAM. We then tested which additional metabolic parameters attenuated the linear and quadratic components of the GDM risk, using just a subset of the samples for whom we had HOMA data available.

The Shapiro-Wilk test was used to test for normality. Unless otherwise stated, all other data are presented as means (95\% confidence intervals). Where regression coefficients $(\beta)$ are shown in analyses of continuous variables they are standardised throughout. Statistical analyses were performed using Stata 13 (StataCorp LP, College Station, Texas, U.S.A.). $p<0.05$ was considered statistically significant throughout.

\section{Results}

\section{Characteristics of the study participants}

In this cohort, $96.9 \%$ of the babies were of white ethnicity, $0.8 \%$ were of mixed race, $0.6 \%$ were black (African or Caribbean), $0.8 \%$ were East-Asian, and $0.9 \%$ were Indo-Asian. Only those mothers that returned their completed questionnaires were included in this analysis. Of these the mean AAM was 12.9 years and the median was 13 (interquartile range 12-14) years. The clinical characteristics of those women that were included and those that were excluded from the present analysis due to non-return of their questionnaires are shown in Supplementary Table 1 (Online Resource 1). The unadjusted birth weights were higher in those included in the present analysis (alongside a 2 day older gestational age at birth), although the difference in birth weight disappeared when adjustment was made for gestational age. There were proportionally fewer women that smoked during pregnancy amongst those that were included in this analysis. The distribution of the AAM was normal (Shapiro-Wilk $p=0.9$ ).

\section{Association between age at menarche and gestational diabetes}

Clinical characteristics of those study participants who developed GDM (9.8\%) and those that did not are shown in Supplementary Table 2 (Online Resource 1). There was no significant difference in AAM between those that developed GDM and those that did not. In linear models with AAM as the independent variable there was no association between AAM and later GDM risk [odds ratio (OR) $0.97(0.83,1.13)$ per year, $p=0.7, n=889]$. However, in quadratic models there was a significant U-shaped association between AAM and GDM at week 28 [linear term OR $0.08(0.02,0.36)$ per year, $p=9.5 \times 10^{-4}$; quadratic term OR $1.10(1.04,1.17)$, $p=1.0 \times 10^{-3} ; n=889$; overall model pseudo $\mathrm{r}^{2}=1.7 \%$ and $\left.p=8.1 \times 10^{-3}\right]$. Relative risks for individual AAM categories are shown in Table 1 . Restricting the analyses to the samples for which we had pre-pregnancy BMI data; there was still a U-shaped association between AAM and GDM risk [linear term OR $0.08(0.02-0.40)$ per year, $p=2.0 \times 10^{-3}$; quadratic term OR $1.10(1.03-1.17), p=2.4 \times 10^{-3}$; $n=798$ ]. AAM showed a negative linear relationship with pre-pregnancy BMI $\left(\beta=-0.22, p=6.0 \times 10^{-10}, n=798\right)$ but addition of pregnancy BMI did not attenuate either the linear or quadratic terms of the association between AAM and GDM [adjusted linear term OR $0.08(0.02,0.41)$ per year, $p=2.5 \times 10^{-3}$; quadratic term OR $1.10(1.04,1.18)$, $\left.p=2.1 \times 10^{-3} ; n=798\right]$.

AAM showed negative linear but not quadratic associations with both HOMA IR and B (Table 2). We tested whether these insulin traits attenuate the U-shaped association between AAM and GDM risk in a subset of women for which we had full HOMA data available. In this subset, there was still a significant pre-adjustment U-shaped association between age at menarche and GDM, albeit weaker than in the full set of women due to the smaller sample size [linear term OR $0.14(0.02-0.82)$ per year, $p=0.03, n=771$; quadratic term OR $1.08(1.01-1.16), p=0.04]$. This association was attenuated when adjusting for HOMA IR [linear term OR $0.18(0.03-1.25)$ per year, $p=0.08, n=771$; quadratic term OR 1.07 (0.99-1.15), $p=0.08$, but not when adjusting for HOMA B [linear term OR $0.12(0.02-0.73)$ per year, $p=0.02, n=771$; quadratic term OR 1.08 (1.01-1.16), $p=0.03]$.

\section{Association between age at menarche and indices from the week 28 oral glucose tolerance test}

Summary data from the study participants' OGTTs are shown in Supplementary Table 3 (Online Resource 1). In the full sample set AAM was associated with week 28 fasting glucose concentration in a U-shaped fashion (linear term $\beta=-1.049$,

Table 1 The relative risks of GDM in pregnancy in the Cambridge Baby Growth Study for groups stratified by AAM

\begin{tabular}{llcl}
\hline $\begin{array}{l}\text { Age at } \\
\text { menarche } \\
\text { (years) }\end{array}$ & $\begin{array}{l}\text { Gestational dia- } \\
\text { betes }[n(\%)]\end{array}$ & $\begin{array}{l}\text { Not gestational } \\
\text { diabetes }[n(\%)]\end{array}$ & Relative risk \\
\hline $8-9.9$ & $3(33.3)$ & $6(66.7)$ & $3.7(1.4,9.7)$ \\
$10-11.9$ & $14(9.6)$ & $132(90.4)$ & $1.1(0.6,1.9)$ \\
$12-13.9$ & $42(9.1)$ & $422(90.9)$ & Reference \\
$14-15.9$ & $21(8.8)$ & $217(91.2)$ & $1.0(0.6,1.6)$ \\
$16-17.9$ & $7(21.9)$ & $25(78.1)$ & $2.4(1.2,4.9)$ \\
\hline
\end{tabular}


Table 2 Linear and quadratic associations between AAM and indices derived from the OGTT (and related variables)

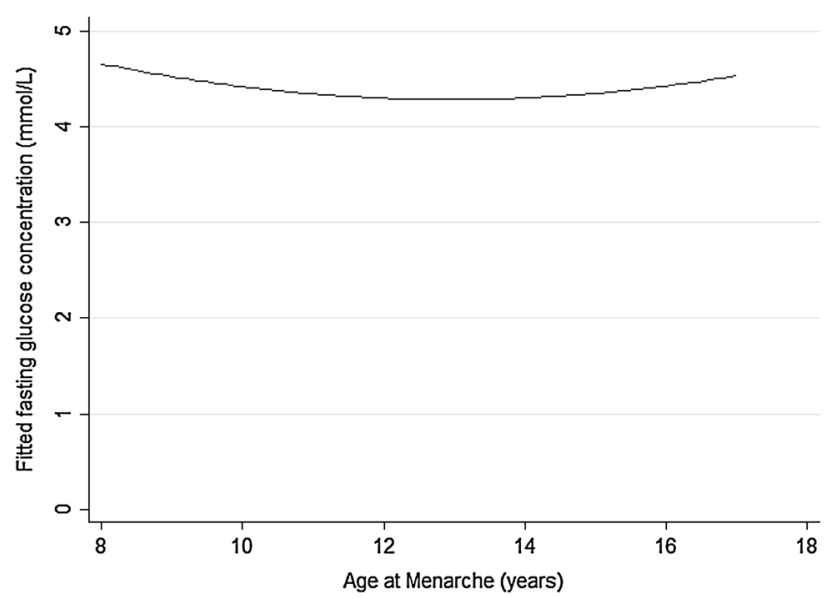

Fig. 1 Fitted fasting glucose concentrations in week 28 of pregnancy by AAM in the Cambridge Baby Growth Study

$p=0.01 ;$ quadratic term $\beta=1.032, p=0.01 ; n=889$ ) (Fig. 1). This relationship was non-significant in the sub-sample restricted to those women where HOMA data were available (linear term $\beta=-0.785, p=0.08$; quadratic term $\beta=0.754$, $p=0.10 ; n=771$ ), and was further weakened when adjusted for HOMA IR (linear term $\beta=-0.443, p=0.29$; quadratic term $\beta=0.465, p=0.27 ; n=771$ ). In contrast it was strengthened when adjusted for HOMA B (linear term $\beta=-0.898$, $p=0.04$; quadratic term $\beta=0.828, p=0.05 ; n=771)$. Unlike with the fasting samples, AAM showed negative linear associations with OGTT 60 min. glucose concentrations (Table 2), which persisted after adjusting for HOMA B $(\beta=-0.100$, $p=5.9 \times 10^{-3}, n=763$ ) but not after adjusting for HOMA IR $(\beta=-0.064, p=0.07, n=763)$.

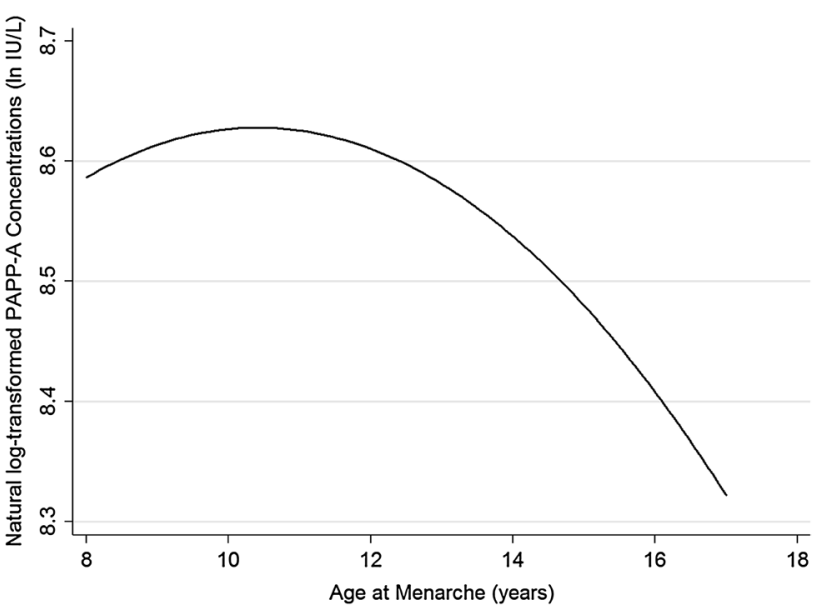

Fig. 2 Fitted (natural log-transformed) PAPP-A concentrations around week 15 of pregnancy by AAM in the Cambridge Baby Growth Study

\section{Association between age at menarche and serum PAPP-A concentrations earlier in pregnancy}

AAM was not linearly related to week 15 serum PAPP-A concentrations $(\beta=-0.014, p=0.7, n=501$; adjusted for the exact stage of gestation when the blood sample was collected). However, there was an inverse U-shaped association between week 15 serum PAPP-A concentrations and AAM (linear term: $\beta=1.807, p=0.02$; quadratic term: $\beta=-1.823$, $p=0.02, n=501$; adjusted as above; Fig. 2).

\section{Discussion}

In this analysis we have confirmed associations between AAM and the subsequent higher risks of GDM. Not surprisingly there were also relationships between AAM and raised glucose concentrations (both fasting and stimulated) 
in pregnancy. Consistent with the findings of $\mathrm{Li}$ et al. [12], the relationship between AAM and risk of GDM in our population was U-shaped. This pattern of relationship has also previously been observed between AAM and type 2 diabetes [22], a condition closely related to GDM [23]. Both conditions are thought to result from a combination of insulin resistance and inadequate insulin secretion, with GDM generally just emerging at a younger age because of the additional physiological insulin resistance of pregnancy [23]. Visual inspection of results from other studies investigating links between AAM and GDM risk finds similar relationships to our U-shaped association even if those studies did not formally test for polynomial associations [11-13]. The one published study that failed to find an association between AAM and GDM risk tested only for a linear relationship [15]. However, on inspection of their data there is an apparent U-shaped relationship. Consistent with the study of Schoenaker et al. [11] the relationship that we found in the present study appeared to be independent of BMI. Indeed our data would suggest that the relationship between AAM and GDM risk relates to insulin resistance rather than adiposity.

In all previous studies reporting a significant association, earlier AAM appears to have a larger influence that later AAM on GDM risk [10-13]. Our data are consistent with this pattern. Indeed the earliest AAM group had a nearly fourfold higher risk of GDM relative to the median AAM groups, albeit that this was modelled from only a small number of participants. The small number of participants in this analysis with the earliest AAM may have led to an inflated relative risk of GDM and the true risk for this group may be closer to the lower end of the $95 \%$ confidence interval. Indeed for AAM of $<10$ years the risk of GDM by meta-analysis of our data combined with that of the other published studies [10-12,15], relative to those with an AAM of 13 years, had an OR of 1.8 with a 95\% confidence interval of 1.6-2.0 (unpublished observation) which is only slightly higher than that reported for an AAM of $<11$ years in a recently published meta-analysis in this area, which of course did not include data from the present study [24]. In our study and those other studies showing a U-shaped relationship between AAM and GDM $[11,12,15]$ there was also a slight increase in risk for GDM associated with late AAM. This increased risk was not observed in all studies [10, 13], but in our pooled random effects dose response meta-analysis of relevant studies it caused a significant non-linearity term (unpublished observation). The mechanism mediating the association between late AAM and slight increased GDM risk is currently unknown, but could involve changes in the sensitivity or secretion of some of the pregnancy factors described below.

Chen et al. [10] hypothesised that the overall relationship between AAM and GDM risk would be underpinned by changes in concentrations of as yet unspecified hormone(s). Results from the current analysis would suggest that they are likely to be hormones that predominantly alter insulin sensitivity. One such hormone could be PAPP-A since we found an inverse U-shaped relationship between AAM and circulating PAPP-A concentrations around week 15 of pregnancy. Previously we reported that low PAPP-A concentrations at this stage of pregnancy are associated with an increased risk of GDM [17]. PAPP-A concentrations were also associated with reduced third trimester insulin sensitivity, possibly due to the modification of localised IGF bioactivity [25] through its role in cleaving IGF-binding protein 4 [26]. Other hormones that could be involved in mediating the link between AAM and insulin resistant GDM include oestrogens [10] since higher concentrations in women have been linked both to early AAM [27-30] and increased GDM risk (when considered relative to sex hormone binding globulin concentrations [31]). Leptin concentrations have also been associated with both AAM [32] and risk of GDM, independent of adiposity [33, 34]. Finally, in metabolic terms, the link between AAM and GDM risk may also involve triacylglycerols since their circulating concentrations may be related to both AAM [35, 36] and GDM risk [37].

The present analysis has advanced knowledge of the link between AAM and GDM risk in pregnancy by highlighting the mediating role of insulin resistance. Our estimate of pancreatic $\beta$-cell function appeared to have a lesser role in AAM-related GDM risk. It did attenuate the OGTT C-peptide disposition index association with AAM, but this is not surprising for an indicator of insulin secretion (relative to its sensitivity). The strengths of our analysis include its prospective nature, the availability of indices related to the glucose-insulin axis in the third trimester of pregnancy from the OGTT, and HOMA modelling using C-peptide rather than insulin concentrations (the former being unaffected by hepatic extraction). Its limitations include the small number of participants in the analysis at the extremes of AAM, which may have led to an inflation of the relative risks of GDM associated with these AAM categories (although there was still a significant relationship evident between AAM and GDM risk, albeit weakened, if the analysis was restricted to women with an AAM between 10 and 16 years; data not shown). This limitation did not substantially affect the overall relationship between AAM and GDM risk as AAM was modelled primarily as a continuous variable. A further limitation is that the ages at menarche were selfreported which can be inaccurate. However, in other studies, self-reported AAM is thought to be moderately accurate in women with higher educational attainment [38] like the majority of women recruited to the Cambridge Baby Growth Study [39]. Finally, as is common with cohort studies that were not designed explicitly to test each different hypothesis in all of the specific studies they are used for, the number 
of study participants in each of our statistical models varies due to missing data. Whilst this could theoretically introduce a degree of bias into the analyses we have no actual evidence of this and the statistical analyses present a biologically plausible explanation for the association between $\mathrm{AAM}$ and GDM risk.

In conclusion this study has confirmed the previously observed U-shaped association between AAM and future GDM risk [10-13] that is independent of pre-pregnancy BMI and therefore adiposity [11]. Our findings add, for the first time, that insulin resistance appears to mediate this relationship. Future studies should continue to investigate the possible hormonal mechanism(s) linking age at menarche to insulin resistance and subsequent risk of GDM.

Data availability The datasets generated during and/or analysed during the current study are available here: https://doi. org/10.17863/CAM.18259.

Acknowledgements The authors thank all the families that took part in the Cambridge Baby Growth Study, and we acknowledge the crucial role played by the research nurses especially Suzanne Smith, AnnMarie Wardell and Karen Forbes, staff at the Addenbrooke's Wellcome Trust Clinical Research Facility, and midwives at the Rosie Maternity Hospital. Funding for this study has come from the Wellbeing of Women (the Royal College of Obstetricians and Gynaecologists, UK) (RG1644). Other core funding has come from the Medical Research Council (7500001180, G1001995, U106179472), European Union Framework 5 (QLK4-1999-01422), the Mothercare Charitable Foundation (RG54608), Newlife Foundation for Disabled Children (07/20), and the World Cancer Research Fund International (2004/03). In addition, there has been support from National Institute for Health Research Cambridge Biomedical Research Centre. KO is supported by the Medical Research Council (Unit Programme number: MC_UU_12015/2).

\section{Compliance with ethical standards}

Conflict of interest The authors declare that they have no conflict of interest.

Statement of human and animal rights All procedures followed were in accordance with the ethical standards of the responsible committee on human experimentation (institutional and national) and with the Helsinki Declaration of 1975, as revised in 2008.

Statement of informed consent Informed consent was obtained from all patients for being included in the study.

Open Access This article is distributed under the terms of the Creative Commons Attribution 4.0 International License (http://creativeco mmons.org/licenses/by/4.0/), which permits unrestricted use, distribution, and reproduction in any medium, provided you give appropriate credit to the original author(s) and the source, provide a link to the Creative Commons license, and indicate if changes were made.

\section{References}

1. Papadimitriou A (2016) The evolution of the age at menarche from prehistorical to modern times. J Pediatr Adolesc Gynecol 29:527-530. https://www.sciencedirect.com/science/article/pii/ S1083318815004295

2. DiVall SA, Radovick S (2008) Pubertal development and menarche. Ann N Y Acad Sci 1135:19-28. https://onlinelibrary. wiley.com/doi/10.1196/annals.1429.026/abstract

3. Ahmed ML, Ong KK, Dunger DB (2009) Childhood obesity and the timing of puberty. Trends Endocrinol Metab 20:237-242. https://www.sciencedirect.com/science/article/pii/S104327600 9000587

4. Prentice P, Viner RM (2013) Pubertal timing and adult obesity and cardiometabolic risk in women and men: a systematic review and meta-analysis. Int J Obes (Lond) 37:1036-1043. https://www.nature.com/articles/ijo2012177

5. Janghorbani M, Mansourian M, Hosseini E (2014) Systematic review and meta-analysis of age at menarche and risk of type 2 diabetes. Acta Diabetol 51:519-528. https://link.springer.com/ article/10.1007\%2Fs00592-014-0579-X

6. Lieberoth S, Gade EJ, Brok J, Backer V, Thomsen SF (2014) Age at menarche and risk of asthma: systematic review and meta-analysis. J Asthma 51:559-565. https://www.tandfonlin e.com/doi/abs/10.3109/02770903.2014.903966?journalCod $\mathrm{e}=\mathrm{ijas} 20$

7. Nnoaham KE, Webster P, Kumbang J, Kennedy SH, Zondervan KT (2012) Is early age at menarche a risk factor for endometriosis? A systematic review and meta-analysis of case-control studies. Fertil Steril 98:702-712.e6. https://www.sciencedirect.com/ science/article/pii/S0015028212005894

8. Anderson KN, Schwab RB, Martinez ME (2014) Reproductive risk factors and breast cancer subtypes: a review of the literature. Breast Cancer Res Treat 144:1-10. https://link.springer.com/artic le/10.1007\%2Fs10549-014-2852-7

9. Charalampopoulos D, McLoughlin A, Elks CE, Ong KK (2014) Age at menarche and risks of all-cause and cardiovascular death: a systematic review and meta-analysis. Am J Epidemiol 180:29-40. https://academic.oup.com/aje/article/180/1/29/2739245

10. Chen L, Li S, He C, Zhu Y, Buck Louis GM, Yeung E, Hu FB, Zhang C (2016) Age at menarche and risk of gestational diabetes mellitus: a prospective cohort study among 27,482 women. Diabetes Care 39:469-471. http://care.diabetesjournals.org/conte $\mathrm{nt} / 39 / 3 / 469$.long

11. Schoenaker DAJM., Mishra GD (2017) Association between age at menarche and gestational diabetes mellitus: The Australian Longitudinal Study on Women's Health. Am J Epidemiol 185:554-561. https://academic.oup.com/aje/article/185/7/554/3045901

12. Li H, Shen L, Song L, Liu B, Zheng X, Xu S, Wang Y (2017) Early age at menarche and gestational diabetes mellitus risk: results from the Healthy Baby Cohort study. Diabetes Metab 43:248-252. http://www.em-consulte.com/article/1103009

13. Shen Y, Hu H, Taylor BD, Kan H, Xu X (2017) Early menarche and gestational diabetes mellitus at first live birth. Matern Child Health J 21:593-598. https://link.springer.com/artic le/10.1007\%2Fs10995-016-2143-5

14. Loffer FD (1975) Decreased carbohydrate tolerance in pregnant patients with an early menarche. Am J Obstet Gynecol 123:180 184. https://www.sciencedirect.com/science/article/pii/00029 37875905244

15. Dishi M, Enquobahrie DA, Abetew DF, Qiu C, Rudra CB, Williams MA (2011) Age at menarche, menstrual cycle characteristics and risk of gestational diabetes. Diabetes Res Clin Pract 93:437-442. https://www.sciencedirect.com/science/article/pii/ S0168822711003512 
16. Farina A, Eklund E, Bernabini D, Paladino M, Righetti F, Monti G, Lambert-Messerlian G (2017) A first-trimester biomarker panel for predicting the development of gestational diabetes. Reprod Sci 24:954-959. http://journals.sagepub.com/doi/abs/10.1177/19337 19116675057

17. Petry CJ, Ong KK, Hughes IA, Acerini CL, Frystyk J, Dunger DB (2017) Early pregnancy-associated plasma protein a concentrations are associated with third trimester insulin sensitivity. J Clin Endocrinol Metab 102:2000-2008. https://academic.oup.com/ jcem/article/102/6/2000/3067658

18. Petry CJ, Mooslehner K, Prentice P, Hayes MG, Nodzenski M, Scholtens DM, Hughes IA, Acerini CL, Ong KK, Lowe WL Jr, Dunger DB (2017) Associations between a fetal imprinted gene allele score and late pregnancy maternal glucose concentrations. Diabetes Metab 43:323-331. http://www.em-consulte.com/artic le/1147465

19. International Association of Diabetes and Pregnancy Study Groups Consensus Panel, Metzger BE, Gabbe SG, Persson B, Buchanan TA, Catalano PA, Damm P, Dyer AR, Leiva Ad, Hod M, Kitzmiler JL, Lowe LP, McIntyre HD, Oats JJ, Omori Y, Schmidt MI (2010) International association of diabetes and pregnancy study groups recommendations on the diagnosis and classification of hyperglycemia in pregnancy. Diabetes Care 33:676-682. http://care.diabetesjournals.org/content/33/3/676. long

20. Lu L, Koulman A, Petry CJ, Jenkins B, Matthews L, Hughes IA, Acerini CL, Ong KK, Dunger DB (2016) An unbiased lipidomics approach identifies early second trimester lipids predictive of maternal glycemic traits and gestational diabetes mellitus. Diabetes Care 39:2232-2239. http://care.diabetesjournals.org/conte nt/39/12/2232.long

21. Levy JC, Matthews DR, Hermans MP (1998) Correct Homeostasis Model Assessment (HOMA) Evaluation uses the computer program. Diabetes Care 21:2191-2192. http://care.diabetesjo urnals.org/content/21/12/2191. Available online at https://www. dtu.ox.ac.uk/homacalculator/

22. Elks CE, Ong KK, Scott RA, van der Schouw YT, Brand JS, Wark PA, Amiano P, Balkau B, Barricarte A, Boeing H, Fonseca-Nunes A, Franks PW, Grioni S, Halkjaer J, Kaaks R, Key TJ, Khaw KT, Mattiello A, Nilsson PM, Overvad K, Palli D, Quirós JR, Rinaldi S, Rolandsson O, Romieu I, Sacerdote C, Sánchez MJ, Spijkerman AM, Tjonneland A, Tormo MJ, Tumino R, van der A DL, Forouhi NG, Sharp SJ, Langenberg C, Riboli E, Wareham NJ; InterAct Consortium (2013) Age at menarche and type 2 diabetes risk: the EPIC-InterAct study. Diabetes Care 36:3526-3534. http://care. diabetesjournals.org/content/36/11/3526.long

23. Petry CJ (ed) (2014) Gestational diabetes: origins, complications and treatment. CRC Press, Boca Raton

24. Sun X, Yang L, Pan J, Yang H, Wu Y, Chen Z, Chen X, Mu L (2018) Age at menarche and the risk of gestational diabetes mellitus: a systematic review and meta-analysis. Endocrine. https:// doi.org/10.1007/s12020-018-1581-9. https://link.springer.com/ article/10.1007\%2Fs12020-018-1581-9

25. Conover CA (2012) Key questions and answers about pregnancy associated plasma protein-A. Trends Endocrinol Metab 23:242249. https://www.sciencedirect.com/science/article/pii/S1043 276012000355

26. Gyrup C, Christiansen M, Oxvig C (2007) Quantification of proteolytically active pregnancy-associated plasma protein-A with an assay based on quenched fluorescence. Clin Chem 53:947-954. http://clinchem.aaccjnls.org/content/53/5/947.long
27. Apter D, Reinilä M, Vihko R (1989) Some endocrine characteristics of early menarche, a risk factor for breast cancer, are preserved into adulthood. Int J Cancer 44:783-787. http://onlin elibrary.wiley.com/doi/10.1002/ijc.2910440506/abstract

28. Emaus A, Espetvedt S, Veierød MB, Ballard-Barbash R, Furberg AS, Ellison PT, Jasienska G, Hjartåker A, Thune I (2008) 17-Beta-estradiol in relation to age at menarche and adult obesity in premenopausal women. Hum Reprod 23:919-927. https://acade mic.oup.com/humrep/article/23/4/919/620597

29. Kirchengast S, Hartmann B (1994) The impact of the age at menarche on body build and sex hormone levels in healthy adult women from Vienna, Austria Anthropol 32:205-214. http://puvod ni.mzm.cz/Anthropologie/article.php?ID=1779

30. Farland LV, Mu F, Eliassen AH, Hankinson SE, Tworoger SS, Barbieri RL, Dowsett M, Pollak MN, Missmer SA (2017) Menstrual cycle characteristics and steroid hormone, prolactin, and growth factor levels in premenopausal women. Cancer Causes Control 28:1441-1452. https://link.springer.com/article/10.1007\%2Fs10 552-017-0971-2

31. Bartha JL, Comino-Delgado R, Romero-Carmona R, Gomez-Jaen MC (2000) Sex hormone binding globulin in gestational diabetes. Acta Obstet Gynecol Scand 79:839-845. http://onlinelibrary.wiley .com/doi/10.1034/j.1600-0412.2000.079010839.x/abstract

32. Matkovic V, Ilich JZ, Skugor M, Badenhop NE, Goel P, Clairmont A, Klisovic D, Nahhas RW, Landoll JD (1997) Leptin is inversely related to age at menarche in human females. J Clin Endocrinol Metab 82:3239-3245. https://academic.oup.com/jcem/ article/82/10/3239/2823183

33. Maghbooli Z, Hossein-Nezhad A, Rahmani M, Shafaei AR, Larijani B (2007) Relationship between leptin concentration and insulin resistance. Horm Metab Res 39:903-907. https://www.thiem e-connect.com/DOI/DOI?10.1055/s-2007-992812

34. Qiu C, Williams MA, Vadachkoria S, Frederick IO, Luthy DA (2004) Increased maternal plasma leptin in early pregnancy and risk of gestational diabetes mellitus. Obstet Gynecol 103:519 525. https://journals.lww.com/greenjournal/Fulltext/2004/03000 /Increased_Maternal_Plasma_Leptin_in_Early.19.aspx

35. Feng Y, Hong X, Wilker E (2008) Effects of age at menarche, reproductive years, and menopause on metabolic risk factors for cardiovascular diseases. Atherosclerosis 196:590-597. https:// www.sciencedirect.com/science/article/pii/S0021915007004029

36. Heys M, Schooling CM, Jiang C, Cowling BJ, Lao X, Zhang W, Cheng KK, Adab P, Thomas GN, Lam TH, Leung GM (2007) Age of menarche and the metabolic syndrome in China. Epidemiology 18:740-746. https://journals.lww.com/epidem/fullt ext/2007/11000/Age_of_Menarche_and_the_Metabolic_Syndr ome_in.15.aspx

37. White SL, Pasupathy D, Sattar N, Nelson SM, Lawlor DA, Briley AL, Seed PT, Welsh P, Poston L; UPBEAT Consortium (2017) Metabolic profiling of gestational diabetes in obese women during pregnancy. Diabetologia 60:1903-1912. https://link.springer.com/ article/10.1007\%2Fs00125-017-4380-6

38. Cooper R, Blell M, Hardy R, Black S, Pollard TM, Wadsworth ME, Pearce MS, Kuh D (2006) Validity of age at menarche selfreported in adulthood. J Epidemiol Commun Health 60:993-997. http://jech.bmj.com/content/60/11/993.long

39. Prentice P, Acerini CL, Eleftheriou A, Hughes IA, Ong KK, Dunger DB (2016) Cohort profile: the Cambridge Baby Growth Study (CBGS). Int J Epidemiol 45:35.a-g. https://academic.oup. com/ije/article/45/1/35/2363713 